\title{
Optimal reconfiguration and capacitor placement for power loss reduction of distribution system using improved binary particle swarm optimization
}

\author{
Mostafa Sedighizadeh • Marzieh Dakhem • \\ Mohammad Sarvi · Hadi Hosseini Kordkheili
}

Received: 18 May 2013/Accepted: 15 November 2013/Published online: 14 March 2014

(C) The Author(s) 2014. This article is published with open access at Springerlink.com

\begin{abstract}
Optimal reconfiguration and capacitor placement are used to reduce power losses and keep the voltage within its allowable interval in power distribution systems considering voltage, current, and radial condition constraints. It is needed to solve two nonlinear discrete optimization problems simultaneously, so an intelligent algorithm is used to reach an optimum solution for network power losses. An effective method and a new optimization algorithm using "improved binary PSO" is presented and discussed to minimize power losses in distribution network by simultaneous networking of reconfiguration and capacitor placement. The proposed model uses binary strings which represent the state of the network switches and capacitors. The algorithm is applied and tested on 16and 33-bus IEEE test systems to find the optimum configuration of the network with regard to power losses. Five different cases are considered and the effectiveness of the
\end{abstract}

M. Sedighizadeh $(\bowtie)$

Faculty of Electrical and Computer Engineering, Shahid

Beheshti University, G. C., Tehran, Iran

e-mail: m_sedighi@sbu.ac.ir

M. Dakhem · M. Sarvi

Faculty of Engineering and Technology, Imam Khomeini

International University, Qazvin, Iran

H. H. Kordkheili

School of Electrical, Electronics and Robotics Engineering,

Shahrood University of Technology, Shahrood, Iran

H. H. Kordkheili

Mazandaran Electric Distribution Co., Mazandaran, Iran proposed technique is also demonstrated with improvements in power loss reduction compared to other previously researched methods, through MATLAB under steady-state conditions.

Keywords Optimal capacitor placement - Optimal reconfiguration - Power loss reduction - Power distribution network $\cdot$ Improved binary particle swarm optimization

\section{Introduction}

Power distribution network consists of a group of radial feeders which can be connected together by several tieswitches and tie-lines. The power loss reduction in the network is a major concern of electric distribution utilities. Among conventional methods, optimal reconfiguration and capacitor placement are two effective methods which can be applied on the network.

The optimization methods have been used to find the optimal location and size of different devices such as capacitors, FACTS, Distributed generations, etc. in power distribution systems [1-3]. In this paper the optimization methods are used for optimal reconfiguration and capacitor allocation in power systems.

Feeder reconfiguration is the process of changing the distribution network topology by changing the status of its switches. There are two kinds of switches based on their open or close conditions: normally open (NO) and normally close (NC). Normally open switches are considered as tie switches in the network. During reconfiguration process, the status of these switches will be changed optimally based on the proposed objective function. Opening and closing of these switches can change the amount of power losses. 
On the other hand, capacitors are mostly used for reactive power compensation in distribution networks. They are also used for power loss reduction and improvement of voltage profiles. The advantages of this kind of compensation depend on how and where to place the capacitors in the network. In recent years, according to proper results of optimal capacitor placement and optimal distribution network reconfiguration for power losses reduction, the idea of using these two methods simultaneously has been considered to maximize the amount of power loss reduction. The combination of these approaches makes the optimization process more complex.

Optimal capacitor placement and feeder reconfiguration have been investigated in many papers separately and various approaches have been used with different objective functions to model the network and optimize the problem solution.

In [4], the branch and bound heuristic method has been used to solve the reconfiguration problem with minimum power losses. In this method, all network switches become closed and will be opened one by one to obtain a new radial configuration. In [5], the power loss reduction and load balancing reconfiguration problem is formulated as an integer programming method. The branch exchange heuristic method is proposed in [6]. In this method, power losses reduction is obtained by closing a switch and opening another one. In [7], an algorithm has been proposed to obtain switch pattern as a function of time. A load flow method based on a heuristic algorithm has been introduced in [8] to determine a configuration with minimal power losses. An implementation using a genetic algorithm (GA) has been proposed in [9]. An improved genetic algorithm based on fuzzy multi-objective approach has been suggested in [10] to solve the problem. In [11], a method based on binary particle swarm optimization (BPSO) algorithm is presented to balance network loads. In [12], the authors have used ant colony search algorithm (ACSA) to solve the reconfiguration problem. Moreover, they have performed a comparison which shows better results for ACSA compared to genetic algorithm (GA) and simulated annealing (SA). A solution procedure employing SA is proposed in [13] and [14] to search for an acceptable non-inferior solution. In [15], the network reconfiguration problem has been solved by a harmony search algorithm (HSA) and an optimal switching combination in the network is obtained which results in minimum power loss.

Many investigations have also been performed to solve the optimal capacitor placement problem. Grainger and Lee [16-18] formulated the problem using a non-linear programming model and solved it by simple iterative procedures based on gradient search. In [19] and [20], the capacitor placement problem is modeled as a mixed integer non-linear programming problem and is solved using a decomposition method and a power flow algorithm. In [21], Tabu search (TS) algorithm is used and a sensitivity analysis is performed to reduce the search space. It is shown that TS has a better performance compared to SA. In [22], the placement problem is solved together with finding the amount of proper capacitors. An approach using GA and a new type of sensitivity analysis method is used. In [23], the implementation of integrated evolutionary algorithms is investigated for solving the capacitor placement optimization problem with reduced annual operating cost. Differential evolution and pattern search (DE-PS) are used as meta-heuristic optimization tools to solve optimal capacitor placement problem.

Some papers considered these two problems simultaneously. In [24], optimal capacitor placement and reconfiguration problem is considered as two consecutive stages and the branch exchange method is used for reconfiguration process. In [25], SA is used to solve optimal capacitor placement and reconfiguration. The amounts of capacitances are determined by a discrete optimization algorithm. Simultaneous solution of these two problems has been approached with the objective of power loss minimization in [26] and the ACSA has been compared to SA and GA and its superior results have been presented. In [27], a modified PSO algorithm is used to solve the optimal capacitor placement and reconfiguration problems. The proposed objective function contains power loss minimization and cost reduction in specific periods of time. In [28], a heuristic algorithm is used for reliability evaluation considering protection schemes and isolation points in the distribution network. The problems are solved by Harmony Search Algorithm. In [29], a deterministic approach for network reconfiguration and a heuristic technique for optimal capacitor placement for power loss reduction and voltage profile improvement in distribution networks are presented. A minimum spanning tree (MST) algorithm is utilized to determine the configuration of minimum power losses along with a GA for optimal capacitor placement.

All previous reports, regardless of their objective function, are looking for the most optimum or a global optimum solution. Meanwhile, each proposed method tries to reach a more optimal or near optimal solution compared to previous works.

In this paper, some planning issues for the priority of reconfiguration and capacitor placement problems in power distribution networks are investigated based on a new improved binary PSO (IBPSO) algorithm. The algorithm is used to solve the simultaneous reconfiguration and optimal capacitor placement problem. The proposed method employs a different structure for the optimization algorithm (by using logical operators AND, OR, and XOR). A nearglobal optimum feeder reconfiguration and capacitor setting is obtained which shows the best results among other 
previous similar works. The proposed IBPSO algorithm is compared with other intelligent algorithms [SA, GA, ant colony optimization (ACO), and ACSA] and its better performance is presented. Then the priority of reconfiguration and capacitor placement cases are presented in the "Discussion" section.

\section{Problem formulation}

The objective is to minimize the network power losses considering constraints under a specified load pattern. The mathematical model of the problem can be expressed as follows [26]:

$\operatorname{Min} F=\operatorname{Min}\left(P_{\mathrm{T}, \text { Loss }}+\lambda_{\mathrm{V}} \times S_{\mathrm{CV}}+\lambda_{\mathrm{I}} \times S_{\mathrm{CI}}\right)$,

where $P_{\mathrm{T}, \mathrm{Loss}}$ is the total real power loss of the network. The parameters $\lambda_{\mathrm{V}}$ and $\lambda_{\mathrm{I}}$ are penalty constants, $S_{\mathrm{CV}}$ is squared sum of the violated voltage constraints, and $S_{\mathrm{CI}}$ is squared sum of the violated current constraints [26]. $\left(\lambda_{\mathrm{V}} \times S_{\mathrm{CV}}+\lambda_{\mathrm{I}} \times S_{\mathrm{CI}}\right)$ is used to apply constraints on the objective function. The voltage magnitude at each bus must remain within its allowable interval. Besides, the current of each branch must satisfy the branch current limitations. These intervals are expressed as follows:

$U_{\min } \leq\left|U_{i}\right| \leq U_{\max }$

$\left|I_{i}\right| \leq I_{i, \max }$

where $\left|U_{i}\right|$ is the voltage magnitude of bus I and $U_{\min }$ and $U_{\max }$ are minimum and maximum allowable voltages, respectively, which are considered as $U_{\min }=0.95 \mathrm{pu}$ and $U_{\max }=1.05 \mathrm{pu} .\left|I_{i}\right|$ is the current magnitude and $I_{i, \max }$ is the maximum current limit of the branch $i$. So, the penalty constants are determined as follows:

1. $\lambda_{\mathrm{V}}$ and/or $\lambda_{\mathrm{I}}$ equal to 0 , if the associated voltage/ current constraint is not violated.

2. A significant value is given to $\lambda_{\mathrm{V}}$ and/or $\lambda_{\mathrm{I}}$ when the associated voltage/current constraint is violated; this makes the objective function move away from the undesirable solution [26]. This significant value is considered equal to the amount of objective function before the optimization process (in this study, the initial power loss of the test network) multiplied by a large number (for example $10^{4}$ ). This process is performed automatically by the developed program.

To obtain the network's power losses and voltage drops, a load flow calculation should be performed during the optimization process. Conventional methods of load flow are Gauss-Seidel and Newton-Rafson which lead to unreliable solutions in radial distribution networks due to the radial structure and the high $R / X$ ratio. Therefore, a backward-forward load flow method (BF-load flow) is used in this paper. Other previous reports chose the same load flow method, too $[27,33]$. The BF-load flow method is fully presented in [32] which is explained briefly in the following section.

\section{Backward-forward (BF) load flow method}

A BF-load flow has been used to evaluate the objective function of the problem. In BF-load flow, the first step is to introduce the topology of distribution network to the computer program as meaningful matrices using graph theory [32]. Then the main process will begin as follows:

1. The vector $\left(U n_{1 \times n}\right)$ containing the voltages of the nodes is defined. At first, we assume that all the elements of the vector are equal to $1 \mathrm{pu}$ (or $1.05 \mathrm{pu}$ ) with zero angle.

2. The currents of nodes $\left(\operatorname{In}_{1 \times n}\right)$ is calculated by the following equation:

$\left[I_{\mathrm{L}}(i)\right]=\left[S_{\mathrm{L}}(i) / U(i)\right]^{*}$,

where $I_{\mathrm{L}}(i), S_{\mathrm{L}}(i)$ and $U(i)$ are current, apparent power and voltage of the load in the node $\# i$, respectively.

3. The current of the last node is the current of the last branch. So the currents of network branches $\left(J_{1 \times f}\right)$ are calculated starting from the last node to the first one by using Kirchhoff's current law (KCL) (Backward sweep). $f$ is the number of network branches.

4. The voltage drop is calculated by the equation $L U=Z J . Z$ is the impedance and $L U_{1 \times f}$ is the voltage drop of each branch.

5. The voltage of reference node is equal to 1 or $1.05 \mathrm{pu}$. The voltage of the next node is calculated by subtracting the corresponding voltage drop from the voltage of previous node (forward sweep) and $U n_{1 \times n}$ will be updated.

6. The procedure is restarted from step 2 with recently calculated $U n_{1 \times n}$. The convergence condition is

$\Delta U n=U n^{(k)}-U n^{(k-1)}<\epsilon$,

where $K$ is the iteration number and $\in$ is the calculation error (precision).

\section{Improved binary particle swarm optimization}

\section{Overview}

Binary particle swarm optimization (BPSO) algorithms were introduced by Kennedy and Eberhart in 1997. In a standard BPSO algorithm, real numbers are used in the 
optimization process. The proposed algorithm uses a method similar to the standard basic PSO and a random method produces a new swarm. The particles' velocities are calculated using the established relationships in the standard mode. The new amounts of particles' position are 0 or 1 which is retrieved from the comparison of calculated velocity with a random number. This method has several disadvantages. The information of particles obtained from previous iterations is not used in the next iteration and the positions of particles are updated almost randomly which leads to inappropriate results. Therefore, "improved binary particle swarm optimization" (IBPSO) algorithm has been introduced in [30].

Improved binary particle swarm optimization

In IBPSO method, the information of every particle in iterations is used to determine new particles. Thus, if each particle contains $d$ dimensions (variables), in the iteration $k+1$ we will have

$v_{i, d}^{k+1}=w_{1} \otimes\left(\operatorname{pbest}_{i, d}^{k} \oplus x_{i, d}^{k}\right)+w_{2} \otimes\left(\right.$ gbest $\left._{d}^{k} \oplus x_{i, d}^{k}\right)$,

where " $\otimes "$, " $\oplus "$ and " + " denote "XOR", "AND", and "OR" operators, respectively. $w_{1}$ and $w_{2}$ are inertia weight factors which are random binary strings. $v_{i, d}^{k+1}$ is the $d$ th dimension velocity of particle $i$ at iteration $k+1$. pbest ${ }_{i, d}^{k}$ and gbest $_{d}^{k}$ are the $d$ th dimension of the personal best position of particle $i$ until iteration $k$ and the $d$ th dimension of the best position of all particles in the swarm at iteration $k$, respectively. The new position of each particle can be updated by Eq. (7):

$x_{i, d}^{k+1}=x_{i, d}^{k} \oplus v_{i, d}^{k+1}$,

where $x_{i, d}^{k}$ is the $d$ th dimension position of particle $i$ at iteration $k$ [30]. The problem will be formulated based on this optimization process.

\section{Implementation of the optimization algorithm}

Implementation steps of the IBPSO algorithm to solve the optimal reconfiguration and capacitor placement problem are as follows:
1. The inputs of the procedure are: $w_{1}$ and $w_{2}$, swarm size $(P)$, objective function and maximum iterations.

2. To start the procedure, the initial swarm is generated containing random binary strings. Each particle of the swarm indicates the ON/OFF condition of switches, the condition of capacitor installation on each node, and the size of located capacitors. Thus, if the number of decision variables (dimension of the search space) is considered $N$, then the initial swarm will be a $P \times N$ matrix as follows:

$$
S=\left[\begin{array}{c}
S_{11} S_{12} \ldots S_{1 N} \\
S_{21} S_{22} \ldots S_{2 N} \\
S_{31} S_{32} \ldots S_{3 N} \\
\cdot \\
\cdot \\
\cdot \\
S_{P 1} S_{P 2} \ldots S_{P N}
\end{array}\right]_{P \times N}
$$

Each row of $S$ is a particle flying in the search space. A special coding process is used to build the matrix $S$.

As mentioned above, each particle consists of three parts: the ON/OFF condition of switches, the condition of capacitor installation on each node, and the size of located capacitors which are denoted by three variables: switch_condition, cap_condition, and cap_size, respectively.

In the switch_condition, the length of binary string is $r_{1}+r_{2}$ where $r_{1}$ is the number of $\mathrm{NC}$ switches and $r_{2}$ is the number of tie-switches. Every branch is considered as a potential switch which can be open or closed. Open and closed switches are shown by 0 and 1 , respectively.

In the cap_condition, the number of required bits $\left(r_{3}\right)$ equals to the number of system nodes. The installation of a capacitor on a node is shown by 1 . If there is no capacitor on a node, the corresponding bit will be 0 in the string.

In the third part of the string, the cap_size, the length of the string equals to the product of the number of nodes $\left(r_{3}\right)$ and the number of capacitors' encoding bits. For example, $(001)_{2}$ to $(110)_{2}$ is the binary number of capacitor types $1-6$ for 6 different capacitances and the number of bits is 3 . Thus, in a 16-node network, the cap_size will be a 48-bit string.

Finally, cap_size and cap_condition bits are multiplied together to determine the location and the size of the capacitors. Figure 1 shows a string of a typical particle.

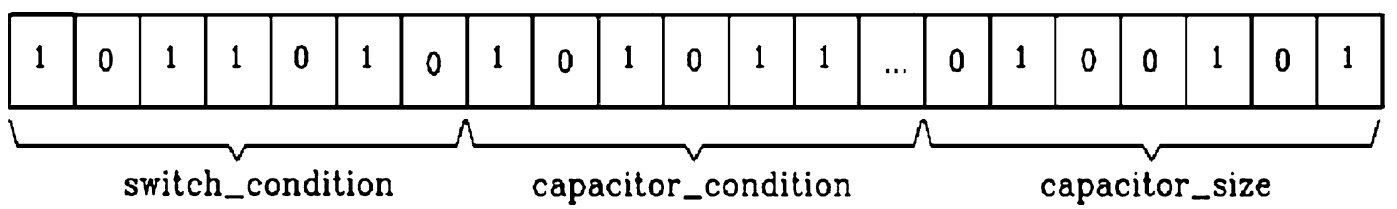

Fig. 1 A string of random typical particle 
Fig. 2 The flowchart of the proposed algorithm

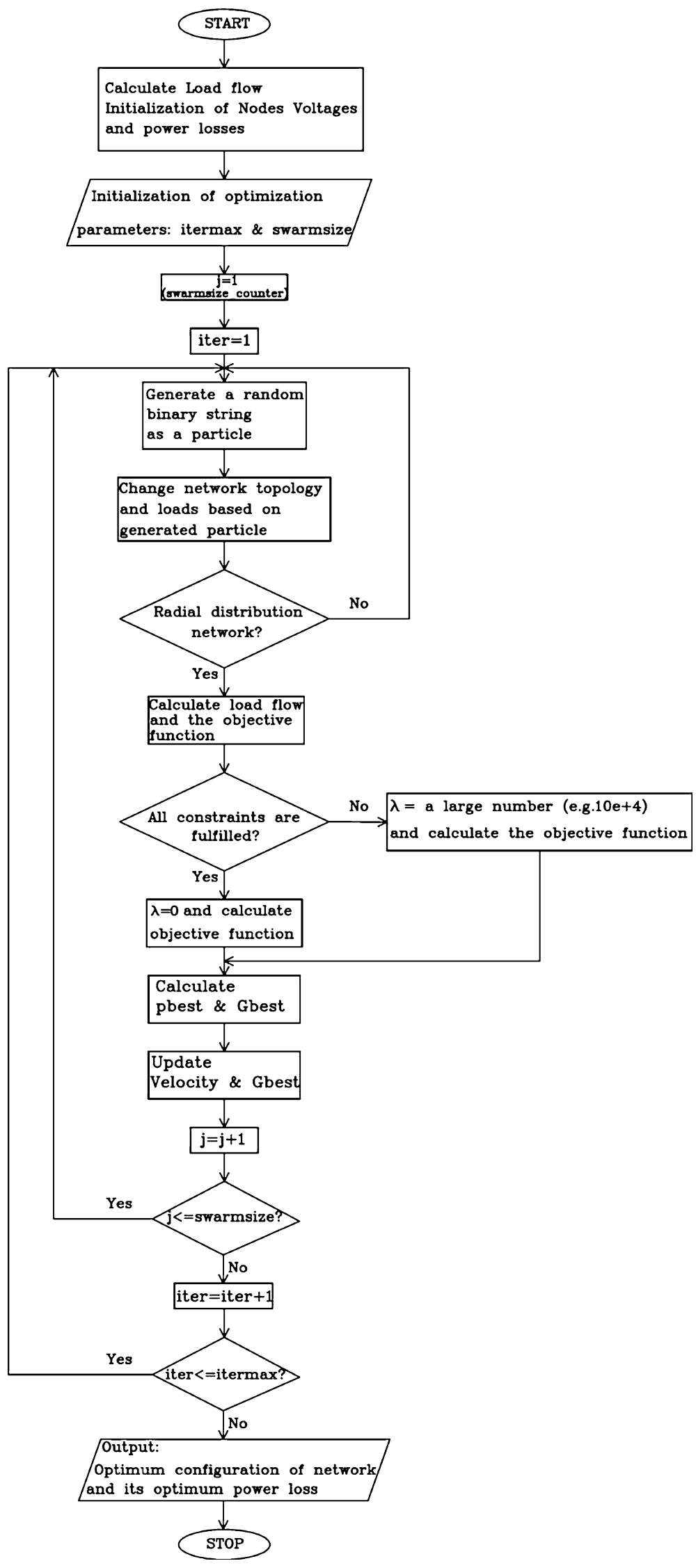


3. Network radial condition is verified in each iteration. This function checks two main conditions: (1) the network must contain no loops; (2) all of the nodes must be energized (connected the supply). So the program considers the radial network as a tree of the initial graph and the above-mentioned condition are verified as the network remains a tree in different states. In other words, a network remains radial if the graph of the network remains a tree. If it is confirmed, then the load flow will be run on the new generated network and the nodes' voltages and the branches currents will be recalculated. In this step, the constraints are also checked and applied to the objective function according to Eq. (1).

4. pbest and gbest are calculated and updated in each iteration. They are the strings which result in the least amount of the objective function. The program will continue to run to reach the maximum predefined number of iterations. The last calculated gbest is the best status of the network and its corresponding power loss is the optimum solution.

Figure 2 shows the flowchart of the proposed process.

\section{Simulation results and discussion}

Optimal reconfiguration and capacitor placement is performed using the proposed algorithm on two IEEE test systems. The systems are well-known benchmark systems which are selected to compare the obtained results to previously reported optimization attempts on the same networks. The algorithm has been implemented in MATLAB R2010.

Six types of capacitors $300,600,900,1,200,1,500$, and $1,800 \mathrm{KVAR}$ are used in capacitor placement. The capacitors are numbered from 1 to 6 , respectively, and 0 denotes a node without any capacitor. The selected ranges of capacitors are the same as previous reports for comparison purposes [26, 27]. Their binary equivalent is defined according to Table 1.

16-bus system

The first study is performed on a 16-bus system with 3 feeders which is shown in Fig. 3. Thirteen continuous lines indicate NC switches, while NO switches (tie-switches) 15 , 21 , and 26 are represented by dotted lines. The initial network power loss is $511.44 \mathrm{~kW} \mathrm{[6].}$

The results of the optimization on a 16-bus test system are shown in Table 2. Power loss has been reduced $12.38 \%$ compared to the initial condition of the network. Five capacitors $(1,500,900,1,800,900$, and $900 \mathrm{KVAR})$
Table 1 Binary equivalent of capacitors

\begin{tabular}{ll}
\hline Types of capacitors & Binary equivalent \\
\hline 0: no capacitor & $\left(\begin{array}{lll}0 & 0 & 0\end{array}\right)$ \\
1: $300 \mathrm{KVAR}$ capacitor & $\left(\begin{array}{lll}0 & 0 & 1\end{array}\right)$ \\
2: $600 \mathrm{KVAR}$ capacitor & $\left(\begin{array}{lll}0 & 1 & 0\end{array}\right)$ \\
3: $900 \mathrm{KVAR}$ capacitor & $\left(\begin{array}{lll}0 & 1 & 1\end{array}\right)$ \\
4: $1,200 \mathrm{KVAR}$ capacitor & $\left(\begin{array}{lll}1 & 0 & 0\end{array}\right)$ \\
5: $1,500 \mathrm{KVAR}$ capacitor & $\left(\begin{array}{lll}1 & 0 & 1\end{array}\right)$ \\
6: $1,800 \mathrm{KVAR}$ capacitor & $\left(\begin{array}{lll}1 & 1 & 0\end{array}\right)$ \\
\hline
\end{tabular}

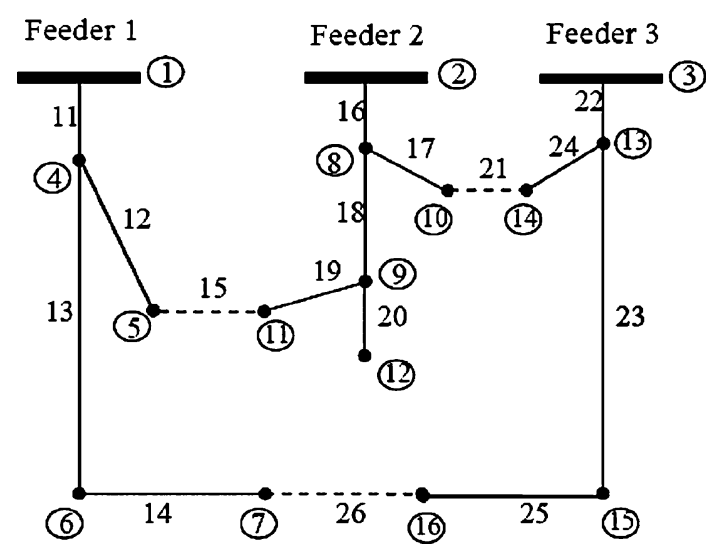

Fig. 3 16-bus distribution system

Table 2 Results of optimal reconfiguration and capacitor placement in 16-bus system

\begin{tabular}{lll}
\hline & $\begin{array}{l}\text { Original } \\
\text { configuration } \\
\text { of system }\end{array}$ & $\begin{array}{l}\text { After reconfiguration } \\
\text { and capacitor placement }\end{array}$ \\
\hline Tie-switches & $15,21,26$ & $19,7,26$ \\
Power loss (KW) & 511.4 & 448.07 \\
Maximum & $U_{\max }=1$ & $U_{\max }=1$ \\
$\quad$ voltage (pu) & (buses 1, 2, 3) & (buses 1, 2, 3) \\
$\begin{array}{c}\text { Minimum } \\
\text { voltage (pu) }\end{array}$ & $\begin{array}{c}U_{\min }=0.9693 \\
\text { (buses 12) }\end{array}$ & $\begin{array}{c}U_{\min }=0.9757 \\
\text { (buses 12) }\end{array}$ \\
$\begin{array}{c}\text { Power loss } \\
\text { reduction (\%) }\end{array}$ & - & 12.38 \\
\hline
\end{tabular}

are used in the optimal case which are placed on buses 4, 7, 8,9 , and 13 , respectively.

Figure 4 shows the voltage profiles before and after optimal reconfiguration and capacitor placement. The voltage profile is obviously improved after the optimization.

\section{3-bus system}

The other case is Baran and $\mathrm{Wu}[19,20]$ test system shown in Fig. 5. The normally open switches (s33, s34, s35, s36, 
Fig. 4 Comparison of voltage profile before and after optimal reconfiguration and capacitor placement in 16-bus test system (dashed line before optimization, solid line after optimization)

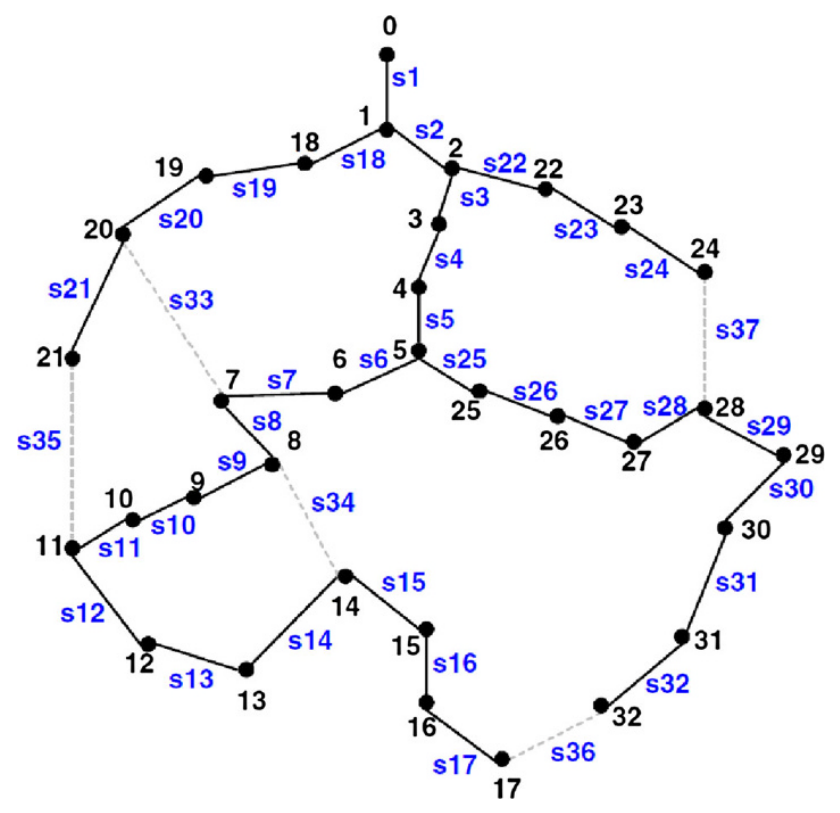

Fig. 5 33-bus test system [19]

s37) are represented by doted lines and normally closed ones (s1-s32) are illustrated by solid lines. The initial power loss is $202.68 \mathrm{~kW}$ [31].

The results of the improved binary PSO algorithm on 33-bus test network are shown in Table 3. Power loss reaches to $93.061 \mathrm{KW}$ which shows a $54.08 \%$ reduction. Five capacitors $(600,300,300,600$, and 300 KVAR) are used in the optimal case which are placed on buses 6,11 , 24, 29, and 32, respectively.
Table 3 Results of optimal reconfiguration and capacitor in 33-bus system

\begin{tabular}{|c|c|c|}
\hline & $\begin{array}{l}\text { Original } \\
\text { configuration } \\
\text { of system }\end{array}$ & $\begin{array}{l}\text { After reconfiguration } \\
\text { and capacitor } \\
\text { placement }\end{array}$ \\
\hline Tie-switches & $33,34,35,36,37$ & $7,9,14,32,37$ \\
\hline Power losses $(\mathrm{KW})$ & 202.68 & 93.061 \\
\hline Maximum voltage (pu) & $\begin{array}{l}U_{\max }=1 \\
\quad \text { (buses 0) }\end{array}$ & $\begin{array}{l}U_{\max }=1 \\
\quad \text { (buses 0) }\end{array}$ \\
\hline Minimum voltage (pu) & $U_{\min }=0.9131$ & $U_{\min }=0.9587$ \\
\hline $\begin{array}{l}\text { Power loss reduction } \\
(\%)\end{array}$ & - & 54.08 \\
\hline
\end{tabular}

Figure 6 shows the voltage profiles before and after the optimal reconfiguration and capacitor placement. Again, it is observed that the voltage profile is improved after applying the optimization.

Discussion

Tables 4 and 5 compare five different cases which employ optimal reconfiguration and capacitor placement separately and simultaneously. The following cases are considered:

Case \#1. Only network reconfiguration;

Case \#2. Only capacitor placement;

Case \#3. First, network reconfiguration and then capacitor placement; 
Fig. 6 Comparison of voltage profile before and after optimal reconfiguration and capacitor placement in 33-bus test system (dashed line before optimization, solid line after optimization)

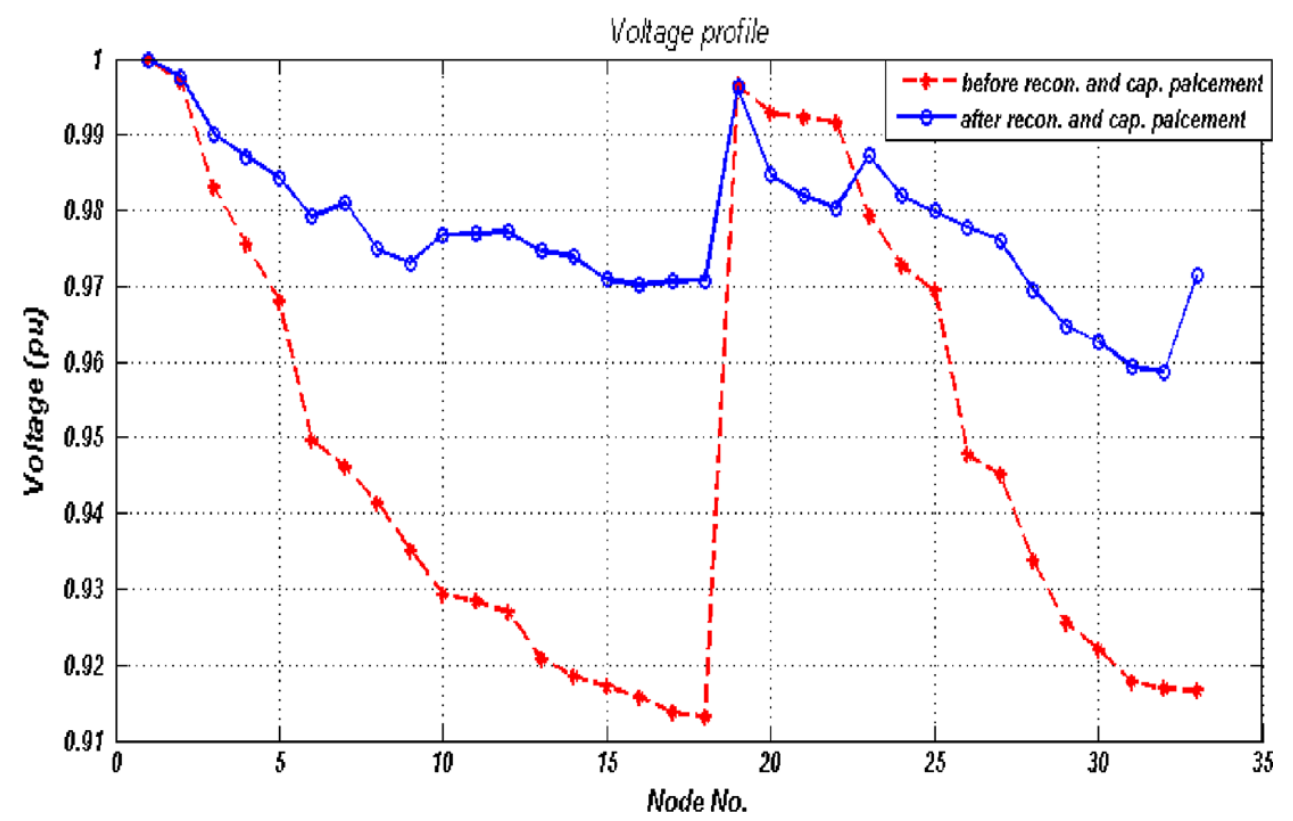

Table 4 comparison of different cases for optimal reconfiguration and capacitor placement in 16-bus system

\begin{tabular}{|c|c|c|c|c|c|c|}
\hline Parameters & Original case & Case 1 & Case 2 & Case 3 & Case 4 & Case 5 \\
\hline \multirow[t]{3}{*}{ Tie-switches } & 15 & 17 & 15 & 17 & 17 & 17 \\
\hline & 21 & 19 & 21 & 19 & 19 & 19 \\
\hline & 26 & 26 & 26 & 26 & 26 & 26 \\
\hline Maximum voltage $(\mathrm{pu})$ & 1 & 1 & 1 & 1 & 1 & 1 \\
\hline Minimum voltage (pu) & 0.9693 & 0.9716 & 0.973 & 0.9763 & 0.9736 & 0.9757 \\
\hline Power losses $(\mathrm{KW})$ & 511.44 & 466.13 & 487.2 & 448.7 & 453.10 & 448.07 \\
\hline Nodes no. & \multicolumn{6}{|c|}{ Placed capacitors (KVAR) } \\
\hline 1 & - & - & - & - & - & - \\
\hline 2 & - & - & - & - & - & - \\
\hline 3 & - & - & - & - & - & - \\
\hline 4 & - & - & 1,800 & 1,200 & 1,800 & 1,500 \\
\hline 5 & - & - & 300 & - & 300 & - \\
\hline 6 & - & - & - & - & - & - \\
\hline 7 & - & - & 900 & 900 & 900 & 900 \\
\hline 8 & - & - & 1,800 & 1,800 & 1,800 & 1,800 \\
\hline 9 & - & - & - & 1,200 & - & 900 \\
\hline 10 & - & - & 1,500 & - & 1,500 & - \\
\hline 11 & - & - & - & - & - & - \\
\hline 12 & - & - & - & - & - & - \\
\hline 13 & - & - & - & - & - & 900 \\
\hline 14 & - & - & - & - & - & - \\
\hline 15 & - & - & - & - & - & - \\
\hline 16 & - & - & - & 300 & - & - \\
\hline
\end{tabular}

Case \#4. First, capacitor placement and then network reconfiguration;

Case \#5. Network reconfiguration and optimal capacitor placement, simultaneously;
Case \#5 (optimal reconfiguration and capacitor placement simultaneously) leads to the most optimum network condition. The comparison is mainly performed between the cases 3, 4, and 5 in which both reconfiguration and capacitor placements 
Table 5 Comparison of different cases for optimal reconfiguration and capacitor placement in 33-bus system

\begin{tabular}{|c|c|c|c|c|c|c|}
\hline Parameters & Original case & Case 1 & Case 2 & Case 3 & Case 4 & Case 5 \\
\hline Tie-switches & $33,34,35,36,37$ & $7,9,14,32,37$ & $33,34,35,36,37$ & $7,9,14,32,37$ & $7,10,34,36,37$ & $7,9,14,32,37$ \\
\hline Maximum voltage (pu) & 1 & 1 & 1 & 1 & 1 & 1 \\
\hline Minimum voltage $(\mathrm{pu})$ & 0.9131 & 0.9378 & 0.9389 & 0.9612 & 0.9658 & 0.9585 \\
\hline Power losses (KW) & 202.68 & 139.55 & 134.2 & 94.26 & 95.91 & 93.06 \\
\hline Nodes no. & \multicolumn{6}{|c|}{ Placed capacitors (KVAR) } \\
\hline 1 & - & - & 900 & - & 900 & - \\
\hline 2 & - & - & - & - & - & - \\
\hline 3 & - & - & 300 & - & 300 & - \\
\hline 4 & - & - & - & 1,200 & - & - \\
\hline 5 & - & - & - & - & - & - \\
\hline 6 & - & - & - & - & - & - \\
\hline 7 & - & - & - & 900 & - & 600 \\
\hline 8 & - & - & - & 1,800 & - & - \\
\hline 9 & - & - & - & 1,200 & - & - \\
\hline 10 & - & - & - & - & - & - \\
\hline 11 & - & - & - & - & - & - \\
\hline 12 & - & - & - & - & - & 300 \\
\hline 13 & - & - & - & - & - & - \\
\hline 14 & - & - & 300 & - & 300 & - \\
\hline 15 & - & - & - & - & - & - \\
\hline 16 & - & - & - & 300 & - & - \\
\hline 17 & - & - & - & - & - & - \\
\hline 18 & - & - & - & - & - & - \\
\hline 19 & - & - & - & - & - & - \\
\hline 20 & - & - & - & - & - & - \\
\hline 21 & - & - & - & - & - & - \\
\hline 22 & - & - & 300 & - & 300 & - \\
\hline 23 & - & - & - & - & - & - \\
\hline 24 & - & - & 300 & - & 300 & - \\
\hline 25 & - & - & - & - & - & 300 \\
\hline 26 & - & - & - & - & - & - \\
\hline 27 & - & - & - & - & - & - \\
\hline 28 & - & - & - & - & - & - \\
\hline 29 & - & - & - & - & - & - \\
\hline 30 & - & - & 600 & - & 600 & 600 \\
\hline 31 & - & - & 600 & - & 600 & - \\
\hline 32 & - & - & - & - & - & - \\
\hline 33 & - & - & - & - & - & 300 \\
\hline
\end{tabular}

are carried out. However, there are differences between priorities of these cases. According to the amount of power loss reduction resulting from the simulation, optimization cases are categorized in distribution networks as follows:

1. Case \#5 (Optimal network reconfiguration and capacitor placement simultaneously);

2. Case \#3 (First, network reconfiguration and then capacitor placement);
3. Case \#4 (First, capacitor placement and then network reconfiguration);

The other conclusion is obtained by comparing the results of IBPSO algorithm to other intelligent methods that were previously applied in different references with similar conditions (constant power loads and the consideration of voltage and current constraints) in 16-bus system. Table 6 shows this comparison. It is concluded that 
Table 6 Comparison of intelligent algorithms to solve optimal reconfiguration and capacitor placement problem in 16-bus system

Table 7 Comparison of intelligent algorithms to solve optimal reconfiguration and capacitor placement problem in 33-bus system

the proposed algorithm in this paper leads to a better result than other previously proposed methods.

The comparison is also done in the 33-bus case. Table 7 compares the result of applied methods to previous studies in a similar condition on the 33-bus system. Significant improvement is obtained using the proposed algorithm compared to previous reports of other researchers.

\section{Environmental benefits}

One of the most important benefits of power and energy loss reduction is its positive environmental impacts. This subject is so important especially in countries like Iran where a large amount of power generation is from fossil fuel power plants.

In such countries, the serious consequence of increase in conventional power generation is the increase of $\mathrm{CO}_{2}, \mathrm{SO}_{x}$ and $\mathrm{NO}_{x}$ emissions. Since the power and energy losses reduce power plants' useful generation, any effective method to decrease the losses is so important. On the other hand, power distribution networks are responsible for a significant percentage of power and energy losses.

The proposed method in this paper is able to decrease the amount of losses in power distribution networks, considerably. In conventional power plants, the generated emissions are equal to $720 \mathrm{~kg} \mathrm{CO}_{2}, 0.1 \mathrm{~kg} \mathrm{NO}_{x}$, and $0.0036 \mathrm{~kg}$ $\mathrm{SO}_{2}$ per MWh. By using the proposed algorithm, the amount of emission can be decreased, consequently [34].

The amount of annual energy loss reduction can be calculated as follows:

energy losses $=8,760 \times$ power losses $\times L_{\mathrm{F}}$,

where $L_{\mathrm{F}}$ is the loss factor. In this study, all loads are considered to be residential with $L_{\mathrm{F}}$ equals to 0.3 .

Table 8 shows the amount of emission reduction due to the reduction of energy losses in the test networks.
Table 8 The amount of emission reduction due to the reduction of energy losses in the test networks by applying optimal reconfiguration and optimal capacitor placement simultaneously

\begin{tabular}{lllll}
\hline & $\begin{array}{l}\text { Annual energy } \\
\text { losses (MWh) }\end{array}$ & $\begin{array}{l}\mathrm{CO}_{2} \\
\text { reduction } \\
(\mathrm{kg})\end{array}$ & $\begin{array}{l}\mathrm{SO}_{2} \\
\text { reduction } \\
(\mathrm{kg})\end{array}$ & $\begin{array}{l}\mathrm{NO}_{x} \\
\text { reduction } \\
(\mathrm{kg})\end{array}$ \\
\hline $\begin{array}{c}\text { 16-bus test } \\
\text { network }\end{array}$ & 166.431 & $119,830.32$ & 0.6 & 16.643 \\
$\begin{array}{c}\text { 33-bus test } \\
\text { network }\end{array}$ & 288.078 & $207,416.16$ & 1.037 & 28.807 \\
\hline
\end{tabular}

It is clear from Table 8 that the amount of $\mathrm{CO}_{2}, \mathrm{SO}_{2}$, and $\mathrm{NO}_{x}$ emissions are significantly decreased.

\section{Conclusion}

In this paper, simultaneous optimal reconfiguration and capacitor placement problem is solved in distribution networks to achieve the minimum power loss in the network. In the optimization process, the applied constraints are voltages of nodes, currents of branches, and radial condition of the network. The minimum power losses with improved voltage profile can be achieved simply. Optimal capacitor placement can control the flow of reactive power in distribution lines which in turn leads to power loss reduction, too. The proposed "improved binary PSO" algorithm has been tested on two 16 and 33 bus networks. It is concluded that simultaneous solving of these two problems will lead to better results than their separated solution. It is also shown that IBPSO leads to better results compared to other previous similar studies using other intelligent methods. Simulations and comparison results show that optimal reconfiguration and capacitor placement simultaneously result in the most optimum condition of network. According to amount of power loss reduction, the priority of optimization cases is proposed as follows which can be used by utilities to plan their networks optimally:(1) optimal network reconfiguration and capacitor placement simultaneously; (2) first, network reconfiguration and then capacitor placement; (3) first, capacitor placement and then network reconfiguration. The power distribution utilities can prioritize their network improvements optimally by using the above results. The environmental benefits of energy losses reduction were briefly presented.

Acknowledgments The authors were supported in part by a research grant from shahidbeheshti university, G. C., the authors are indebted to the editor andreferees for greatly improving this paper.

Authors' contributions MS has given the techniques and ideas of the paper (corresponding author).He also removed technical as well as grammatical mistakes from the paper.MS suggested the good notes for improving paper. HK helped our toanalyze the data using Matlab 
software. MD performed simulations, analyzedthe data, handled all the comments from reviewer side and compiled thepaper by getting suggestions from coauthors. All authors read and approvedthe final manuscript.

Open Access This article is distributed under the terms of the Creative Commons Attribution License which permits any use, distribution, and reproduction in any medium, provided the original author(s) and the source are credited.

\section{References}

1. Kayal, P., Chanda, C.K.: A simple and fast approach for allocation and size evaluation of distributed generation. Int. J. Energy Environ. Eng. 4, 7 (2013)

2. Lal, S., Laturi, A.: Techno-economic analysis of a hybrid minigrid system for Fiji islands. Int. J. Energy. Environ. Eng. 3, 10 (2012)

3. Islam, S.M.: Increasing wind energy penetration level using pumped hydro storage in island micro-grid system. Int. J. Energy. Environ. Eng. 3, 9 (2012)

4. Merlin, A., Back, H.: Search for a minimal loss operating spanning tree configuration for an urban power distribution system. In: Proceedings of the 5th Power System Computation Conference (PSOC), Cambridge, 1975, pp. 1-18 (1975)

5. Baran, M.E., Wu, F.F.: Network reconfiguration in distribution systems for loss reduction and load balancing. IEEE Trans. Power Deliv. 4(2), 1401-1407 (1989)

6. Civanlar, S., Grainger, J.J., Yin, H., Lee, H.: Distribution feeder reconfiguration for loss reduction. IEEE Trans. Power Deliv. 3(3), 1217-1223 (1988)

7. Broadwater, R.P., Khan, A.H., Shalaan, H.E., Lee, R.E.: Time varying load analysis to reduce distribution losses through reconfiguration. IEEE Trans. Power Deliv. 8(1), 294-300 (1993)

8. Shirmohammadi, D., Wayne Hong, H.: Reconfiguration of electric distribution network for resistive line losses reduction. IEEE Trans. Power Deliv. 4(2), 1492-1498 (1989)

9. Nara, K., Shiose, A., Kitagawoa, M., Ishihara, T.: Implementation of genetic algorithm for distribution systems loss minimum reconfiguration. IEEE Trans. Power Syst. 7, 1044-1051 (1992)

10. Huang, Y.-C.: Enhanced genetic algorithm-based fuzzy multiobjective approach to distribution network reconfiguration. Gen Transm. Distrib. IEEE Proc. 149(5), 615-620 (2002)

11. Jin, X., Zhao, J., Sum, Y., Li K., Zhang, B.: Distribution network reconfiguration for load balancing using binary particle swarm optimization. International Conference on power system technology (POWERCON 2004), Singapore, 2004, pp. 507-510 (2004)

12. Su, C.-T., Chung, C.F., Chiou, J.-P.: Distribution network reconfiguration for loss reduction by ant colony search algorithm. Electr. Power Syst. Res. 75(2-3), 190-199 (2005)

13. Chiang, H.-D., Rene, J-.J.: Optimal network reconfiguration in distribution systems: part 1: a new formulation and a solution methodology. IEEE Trans. Power Deliv. 5, 1902-1908 (1990)

14. Chiang, H.-D., Rene, J-.J.: Optimal network reconfiguration in distribution systems: part 2: solution algorithms and numerical results. IEEE Trans. Power Deliv. 5, 1568-1574 (1992)

15. Srinivasa Rao, R., Narasimham, S.V.L., Ramalinga Raju, M., Srinivasa Rao, A.: Optimal network reconfiguration of large-scale distribution system using harmony search algorithm. IEEE Trans. Power Syst. 5, 1080-1088 (2011)

16. Grainger, J.J., Lee, S.H.: Optimum size and location of shunt capacitors for reduction of losses on distribution feeders. IEEE Trans. Power Appar. Syst. 100, 1105-1118 (1981)
17. Lee, S.H., Grainger, J.J.: Optimum placement of fixed and switched capacitors on primary distribution feeders. IEEE Trans. Power Appar. Syst. 100, 345-352 (1981)

18. Grainger, J.J., Lee, S.H.: Capacity release by shunt capacitor placement on distribution feeders: a new voltage-dependent model. IEEE Trans. Power Appar. Syst. 101, 1236-1244 (1982)

19. Baran, M.E., Wu, F.F.: Optimal capacitor placement on radial distribution systems. IEEE Trans. Power Deliv. 4, 725734 (1989)

20. Baran, M.E., Wu, F.F.: Optimal sizing of capacitors placed on a radial distribution system. IEEE Trans. Power Deliv. 4, 735-743 (1989)

21. Huang, Y.C., Yang, H.T., Huang, C.L.: Solving the capacitor placement problem in a radial distribution system using tabu search approach. IEEE Trans. Power Syst. 11(4), 1868-1873 (1996)

22. Sundhararajan, S., Pahwa, A.: Optimal selection of capacitor for radial distribution systems using a genetic algorithm. IEEE Trans. Power Syst. 9(3), 1499-1507 (1994)

23. El-Fergany, A.A.: Optimal capacitor allocations using evolutionary algorithms. IET Gen. Transm. Distrib. 7(6), 593-601 (2013)

24. Peponis, G.J., Papadopulos, M.P., Hatziargyriou, N.D.: Optimal operation of distribution networks. IEEE Trans. Power Syst. 11(1), 59-67 (1996)

25. Dan, J., Baldick, R.: Optimal electric distribution system switch reconfiguration and capacitor control. IEEE Trans. Power Syst. 11(2), 890-897 (1996)

26. Chang, C.F.: Reconfiguration and capacitor placement for loss reduction of distribution systems by ant colony search algorithm. IEEE Trans. Power Syst. 23(4), 1747-1755 (2008)

27. Pooya, R., Mehdi, V.: Distribution system efficiency improvement by reconfiguration and capacitor placement using a modified particle swarm optimization algorithm. IEEE Electrical Power and Energy Conference, 2010, pp. 1-6 (2010)

28. Pooya, R., Mehdi, V., Hajipour, E.: Reconfiguration and capacitor placement in radial distribution systems for loss reduction and reliability enhancement. In: Intelligent system application to power systems (ISAP), 2011, pp. 1-6 (2011)

29. Montoya, D.P., Ramirez, J.M.: Reconfiguration and optimal capacitor placement for losses reduction. In: IEEE/PES, transmission and distribution: Latin America Conference and Exposition, 2012, pp. 1-6 (2012)

30. Xiaohui, Y., Hao, N., Anjun, S., Liang, W., Yanbin, Y.: An improved binary particle swarm optimization for unit commitment problem. Expert Syst. Appl. 36, 8049-8055 (2009)

31. Gomes Jr, F.V., Carneiro, S., Pereira, J.L.R., Vinagre, M.P., Garcia, P.A.N., Araújo, L.R.: A new distribution system reconfiguration approach using optimal power flow and sensitivity analysis for loss reduction. IEEE Trans. Power Syst. 21(4), 1616-1623 (2006)

32. Ehsan, M., Javad, R.: A new method for load flow on radial distribution network. In: 12th Iranian Student Conference on electrical engineering (2009)

33. Kasaei, M.J., Gandomkar, M.: Loss reduction in distribution system with Simultaneous using of capacitor placement and reconfiguration by Ant colony algorithm. In: 24th International Power System Conference (2009)

34. Keshavarz, M., Ranjbar, A.M., Sedighizadeh, M., Sheikhi, A.: Bio-objective function micro grid optimal operation on using a hybrid algorithm. Int. J. Tech. Phys. Probl. Eng. (IJTPE) 5(1), 149-162 (2013). (Iss. 14) 